\title{
A universal equivalent circuit for carbon-based supercapacitors
}

\author{
Stephen Fletcher • Victoria Jane Black • Iain Kirkpatrick
}

Received: 8 October 2013 /Revised: 7 November 2013 / Accepted: 7 November 2013 /Published online: 1 December 2013

(C) Springer-Verlag Berlin Heidelberg 2013

\begin{abstract}
A universal equivalent circuit is proposed for carbon-based supercapacitors. The circuit, which actually applies to all porous electrodes having non-branching pores, consists of a single vertical ladder network in series with an $R C$ parallel network. This elegant arrangement explains the three most important shortcomings of present-day supercapacitors, namely open circuit voltage decay, capacitance loss at high frequency, and voltammetric distortion at high scan rate. It also explains the shape of the complex plane impedance plots of commercial devices and reveals why the equivalent series capacitance increases with temperature. Finally, the construction of a solid-state supercapacitor simulator is described. This device is based on a truncated version of the universal equivalent circuit, and it allows experimenters to explore the responses of different supercapacitor designs without having to modify real supercapacitors.
\end{abstract}

Keywords Equivalent circuit - Supercapacitors · Batteries · Porous electrodes · Electronic devices · Carbon · Impedance . Cyclic voltammetry $\cdot$ Self-discharge

\section{Introduction}

Supercapacitors store energy reversibly by trapping charge carriers at the electrode/solution interface [1-5]. Typically, they do this by means of ion adsorption (double-layer capacitors) or by means of surface-confined redox reactions (pseudo-capacitors). In some electrode materials, such as

This paper is dedicated to the memory of Professor Vladimir Sergeevich Bagotsky (1920-2012)

S. Fletcher $(\bowtie) \cdot$ V. J. Black $\cdot$ I. Kirkpatrick

Department of Chemistry, Loughborough University, Ashby Road,

Loughborough, Leicestershire LE11 3TU, UK

e-mail: stephen.fletcher@Lboro.ac.uk carbon, both types of charge trapping occur at the same time. For this reason, carbon has become the material of choice for many commercial supercapacitors. Among the types of carbon that have been studied in detail are activated carbon (the industry standard) [6-13], various templated carbons [14-23], carbon black [24-27], carbon aerogel [28-32], carbon nanotubes [33-46], and graphene [47-56].

Over the past decade, supercapacitors have grown in popularity due to their high specific power, rapid charge/discharge capability, and long cycle life. Indeed, they have already replaced batteries in some niche applications where pulse power is critical, such as flash memory cards, mobile phones, biomedical devices, and digital cameras. On the larger scale, they are also under active consideration as components for uninterruptible power supplies, for regenerative braking in electric vehicles, for cold-starting internal combustion engines, and for load levelling. With more than a dozen major manufacturers worldwide, the optimization of supercapacitor design is now an area of compelling scientific interest.

To a first approximation, the total capacitance of a supercapacitor varies with the surface area of its electrodes, so carbons containing micropores $(d<2 \mathrm{~nm})$ are a natural choice. However, microporous carbons also exhibit a range of unwanted problems, such as open circuit voltage decay, capacitance loss at high frequency, and voltammetric distortions at high scan rate, which limit the performance of working devices. Accordingly, there is a strong need to understand the physical origin of these deleterious effects.

In recent years, various different experimental techniques have been used to characterize supercapacitors. For example, some researchers have applied small-amplitude techniques such as impedance spectroscopy [57-70], while others have applied large-amplitude techniques such as cyclic voltammetry [71-77]. A few pioneers have even performed computer simulations [78-90]. In spite of these diverse efforts, however, no single model has yet emerged that is capable of rationalizing the whole gamut of experimental results. 
A powerful method of modelling an electrochemical system is to derive its equivalent circuit [91-94]. An equivalent circuit is a conceptual arrangement of linear circuit elements which, if implemented in hardware, would generate the same impedance response (or set of current/voltage curves) as the electrochemical system under investigation. In the present work, we demonstrate, for the first time, that a single, universal, equivalent circuit exists for virtually all carbon-based supercapacitors. Furthermore, this same circuit provides a complete and logically consistent explanation for open circuit voltage decay, capacitance loss at high frequency, and voltammetric distortions at high scan rate. Finally, based on a truncated version of the equivalent circuit, a solid-state supercapacitor simulator is constructed which successfully mimics the behaviour of commercial devices.

\section{Experimental}

Voltammetric experiments were carried out using a $\mu$-Autolab 70282 (type II) potentiostat (Metrohm-Autolab, Eco-Chemie, Utrecht, The Netherlands). The operating software was General Purpose Electrochemical System (GPES), version 4.9 (Eco-Chemie, Utrecht, The Netherlands).

AC impedance measurements were carried out using a Wayne-Kerr Precision Component Analyser, model 6430A (Wayne-Kerr Electronics, Chichester, UK). Equivalent series capacitance, equivalent series resistance, impedance magnitude, and phase angle were all measured over the frequency range $20 \mathrm{~Hz}$ to $500 \mathrm{kHz}$.

Open circuit voltage decay measurements were carried out using a PalmSens EmStat ${ }^{2}$ potentiostat (Palm Instruments BV, Houten, The Netherlands). The software was PSTrace, version 3.06 (Palm Instruments BV, Houten, The Netherlands). All devices were charged to $2.0 \mathrm{~V}$ using a Concept Series HY3003-3 D.C. bench top power supply (Digimess Instruments, Derby, UK). After maintaining this voltage for fixed times (typically in the range 1-20 s), the devices were switched to open circuit and the subsequent voltage decay was monitored.

High-temperature supercapacitors were constructed inhouse using the method of [95]. Briefly, the electrodes were made by a screen printing method (DEK 65 manual screen printer, DEK Printing Machines Ltd, Weymouth, UK) and consisted of three printed layers. The first layer was silver (E1660, Ercon, Wareham, MA, USA) which acted as the current collector, the second layer was graphitic carbon (CHSN8032, Sun Chemical, Norton, UK) to mask the redox chemistry of the silver, and the third layer was an activated carbon (DLC SUPRA 30, Norit, Cambuslang, UK). The spacer was glass fibre, and the electrolyte was butyltrimethylammonium bis(trifluoromethylsulfonyl)imide (Io-li-tec GmbH, Heilbronn, Germany).
Commercial 1-F supercapacitors (Maxwell Technologies BCAP0001, 2.7 V) were purchased directly from the manufacturer. The electrodes were activated carbon, the spacer was cellulose, and the electrolyte was tetraethylammonium tetrafluoroborate in acetonitrile.

\section{General theory of equivalent circuits}

Equivalent circuits are generally constrained by a set of boundary conditions, whose purpose is to limit the number of candidate solutions to the problem at hand. Unfortunately, different boundary conditions are commonly assumed in different disciplines, making cross-disciplinary comparisons problematic. An especially vexing situation arises when comparing results obtained by electrical engineers and electrochemists.

Among electrical engineers, the term "equivalent circuit" generally refers to a simple series combination of two components (such as a resistor and a capacitor) that duplicates the response of a real system at a single specified frequency. (This usage is advocated by the American National Standards Institute (ANSI), for example.) Such a circuit is shown in Fig. 1. In this case, there is no requirement that the equivalent circuit should match the amplitude and phase shift of the real system at any other frequency.

Among electrochemists, the phrase "equivalent circuit" has a very different meaning. It refers to a larger network that duplicates the response of a real system at multiple specified frequencies. Although there is no restriction on the size of such a network, electrochemists habitually apply Occam's razor and so do not insert more components into the network than there are physical phenomena to be explained. Nevertheless, they generally consider a large assortment of networks as candidate solutions and then select the "best" network as the one whose component values minimize the sum of the squared residuals between the theoretical impedance function and the experimental impedance function. By contrast, no such selection process is applied in the engineering case.

The existence of these two different philosophies of equivalent circuit modelling means that published values of "capacitance" must be interpreted with caution. To illustrate this

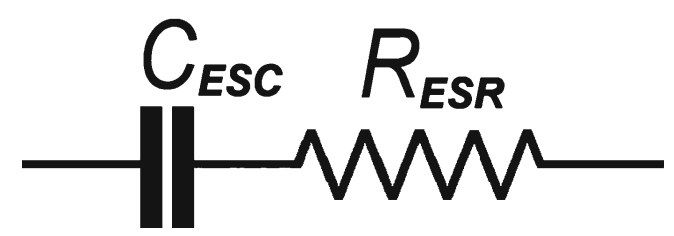

Fig. 1 An example of an engineering equivalent circuit. At each measurement frequency, the capacitance and resistance values are adjusted to match the amplitude and phase response of the real system. The resulting values of $C$ and $R$ are then referred to as "the equivalent series capacitance" (ESC) and "the equivalent series resistance" (ESR) 
point, consider a randomly configured electrical network having a real physical capacitor embedded somewhere inside it. An explicit formula for the capacitance of this capacitor is given by Gauss's law, which states that the stored charge $Q$ is equal to the integral of the normal component of the local electric field $\vec{E}$ (a vector) over the area $\vec{A}$, multiplied by the local absolute permittivity $\varepsilon$.

$Q=\oint_{\varepsilon} \vec{E} \cdot d \vec{A}$

Accordingly, the ratio $Q / V=C_{\text {real }}$ is the real capacitance of interest, which for a parallel plate capacitor of thickness $d$ takes the form

$C_{\text {real }}=\frac{\varepsilon A}{d}=\frac{\varepsilon_{\mathrm{r}} \varepsilon_{0} A}{d}$

where $\varepsilon_{0}$ is the absolute permittivity of the vacuum and $\varepsilon_{\mathrm{r}}$ is the local relative permittivity (dielectric constant) of the medium inside the capacitor.

However, only an electrochemical equivalent circuit could possibly identify $C_{\text {real }}$. An engineering equivalent circuit would fail to do so because its "equivalent series capacitance" is a fictional capacitance whose sole purpose is to mimic the amplitude and phase response of the total system. Thus, the equivalent series capacitance has no simple physical correlative. Indeed, for the equivalent series circuit shown in Fig. 1, it is given by the equation

$C_{\mathrm{ESC}}(\omega)=\frac{1}{j \omega \operatorname{Im} Z(\omega)}$

where $j$ is the square root of minus one, $\omega$ is the angular frequency, and $\operatorname{Im} Z(\omega)$ is the imaginary component of the complex impedance of the total system. Note that, unlike the real physical capacitance, the equivalent series capacitance derived from this formula may be (usually is) frequency dependent. This unphysical result arises because the equivalent series capacitance is compelled to follow the response of the system as a whole (Eq. 3), not just the response of the isolated capacitor (Eq. 2). Consequently,

$$
C_{\mathrm{ESC}}(\omega) \neq \frac{\varepsilon A}{d}
$$

As a result of these considerations, literature plots of capacitance versus "surface area" are meaningless unless the authors have defined exactly what they mean by capacitance and preferably demonstrated that this same capacitance is not frequency dependent.

It is clear from the above discussion that two different approaches to equivalent circuits are in common use, and these generate two different values of capacitance for the same real system. On one hand, there is a two-component ("engineering") equivalent circuit which generates a frequency-dependent equivalent series capacitance whose properties may sometimes violate common sense. On the other hand, there is a multiple-component ("electrochemical") equivalent circuit which generates a frequency-independent real capacitance $\left(C_{\text {real }}\right)$ whose properties are well behaved. It is a matter of regret that, in the scientific literature, these approaches are not always clearly distinguished. A particularly egregious example is the capacitance derived by measuring the baseline hysteresis of voltammograms, which is nearly always an equivalent series capacitance, even though this fact is rarely (if ever) mentioned to the reader. A similar caveat applies to the case of capacitance measurements obtained by bridge methods; these typically generate values of equivalent series capacitance rather than real physical capacitance.

\section{Electrochemical equivalent circuit of carbon-based supercapacitors}

The most obvious method of increasing the real capacitance of carbon electrodes is to increase their internal surface area. However, increasing the internal surface area of any porous solid at fixed volume can be achieved only by decreasing the average diameter of its pores, which necessarily causes an increase in pore resistance. Therefore, when designing a supercapacitor, an important consideration is the trade-off between the total capacitance of the device and the resistance of individual pores.

The modelling of porous solids, in the case of an interface that is blocking to charge transfer, has reached a high level of sophistication [96-101]. The standard model is an array of identical pores arranged in parallel. Each pore, assumed uniform, is then represented by a set of repeated two-port components as shown in Fig. 2. For historical reasons, such an arrangement is called an " $R C$ transmission line". It can be seen that every current pathway through the transmission line passes through a capacitor, so that the impedance approaches infinity at low frequency. This is what we would expect for an

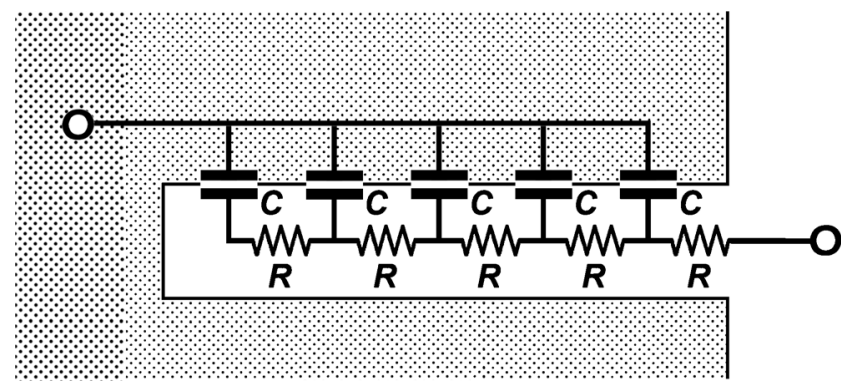

Fig. 2 The RC transmission line model of a single pore. The resistors and capacitors all have the same values, so that the entire transmission line consists of a large number of small repeating sections 
"ideally polarizable" interface. In the scientific literature, transmission line models have been successfully applied to the charge/discharge behaviour of a range of materials containing pores whose diameters are notionally uniform, such as conducting polymers [101], metal oxides in dye-sensitized solar cells [102], and corrosion films [103].

Unfortunately, the RC transmission line model fails completely in the case of materials whose pores are neither identical nor uniform, such as activated carbons. Activated carbons are made by pyrolysis of biological materials such as coconut shell, bituminous coal, and wood. During pyrolysis, the precursor materials expel volatile matter, leaving behind pores having multifarious pore wall chemistries and diameters. In the laboratory, it is commonly found that most of the internal surface area of activated carbon is associated with micropores and mesopores whose diameters fall in the range $0.3-50 \mathrm{~nm}$. Such tiny pores are very difficult to model. In addition, there are often some macropores whose diameters exceed $50 \mathrm{~nm}$.

In 1994 Fletcher [91] published a series of tables of degenerate electrical networks for use in the equivalent circuit analysis of electrochemical systems. (Electrical networks are said to be degenerate if they exhibit identical total impedances at all frequencies.) Among the tabulated results was the one shown in Fig. 3.

Figure 3 is of special interest in the present work because it implies that multiple horizontal RC ladder networks wired in parallel are actually degenerate with a single vertical RC ladder network, given a suitable choice of $R$ and $C$ values.

\section{Horizontal ladder network}

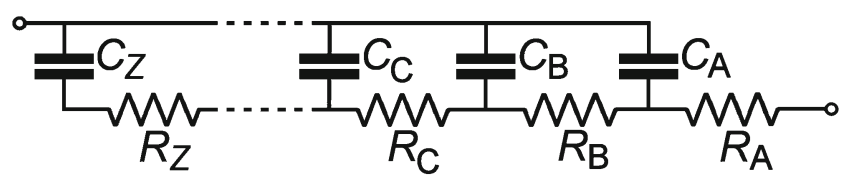

Vertical ladder network

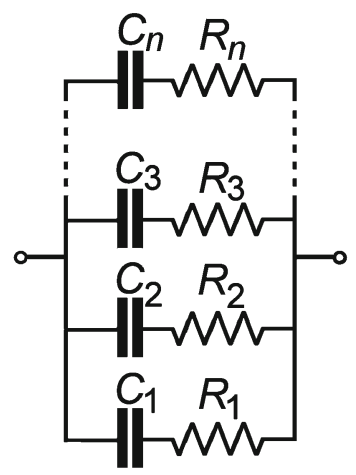

Fig. 3 A horizontal RC ladder network and its vertical equivalent (from [91]). Unlike the simple transmission line, all the components in the horizontal ladder network may have different values. Similarly, all the components in the vertical ladder network may also have different values. Nevertheless, the vertical ladder network can always be made degenerate with the horizontal ladder network by a suitable choice of $R$ and $C$ values
In other words, Fig. 3 implies that any set of non-uniform pores occurring in parallel can be modelled as a vertical ladder network. Thus, the vertical ladder network can be regarded as a universal equivalent circuit for conducting porous solids.

Based on this novel insight, a high-precision equivalent circuit for an assembled symmetric supercapacitor can readily be devised, and this is shown in Fig. 4. It consists of a vertical ladder network of RC-series components, each of which has a different time constant from the others, and a solitary RCparallel network (on the right-hand side of the diagram) which represents the resistance of the bulk of solution shunted by the capacitance (dielectric polarization) of the solution.

At this point, we must caution the reader against a naive interpretation of the model components shown in Fig. 4. While it is certainly true that each capacitor is related to the capacitance of a patch of pore wall, and each resistor is related to the resistance of a length of pore channel, the correspondence is not one-to-one with the real physical values. More precisely, the proposed equivalent circuit is degenerate with another equivalent circuit (a random set of finite-length RC transmission lines in parallel) which does have a one-to-one correspondence with the real physical values. We also caution that the vertical ladder network cannot extend to infinity because it is not possible to have infinite capacitance in a finite space.

In supercapacitor devices, the resistance of the solid carbon is generally insignificant compared with the resistance of the liquid-filled pores, so it is the liquid-filled pores that control the RC time constants in the system. For example, the roomtemperature conductivity of activated carbon is about $0.06 \times$ $10^{3} \mathrm{~S} / \mathrm{cm}$, whereas the room-temperature conductivity of aqueous sulphuric acid at its conductivity maximum (31 wt $\%$ ) is $830 \times 10^{-3} \mathrm{~S} / \mathrm{cm}$. Hence, in this case, the pore

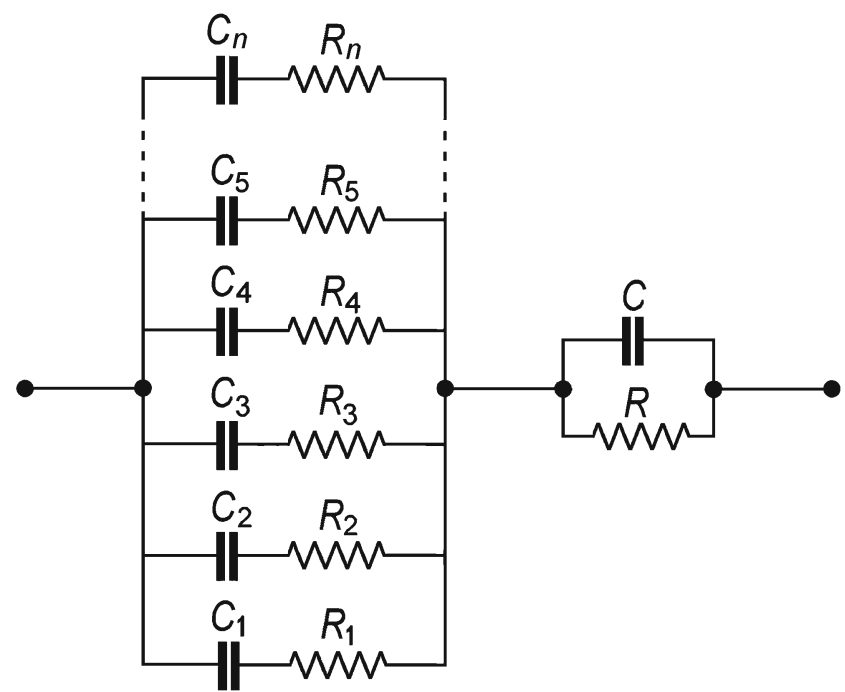

Fig. 4 The universal equivalent circuit for a symmetric carbon-based supercapacitor. It consists of a single vertical ladder network in series with an RC parallel network. (For an asymmetric supercapacitor, two ladder networks would be needed.) 
channels containing aqueous sulphuric acid are more resistive than the pore walls by a factor of circa 70 . The situation is even more extreme in the case of high-temperature supercapacitors [95], which utilize ionic liquids such as butyltrimethylammonium bis(trifluoromethylsulfonyl)imide. This latter compound has a room-temperature conductivity of only $2.1 \times 10^{-3} \mathrm{~S} / \mathrm{cm}$, implying that the pore channels containing ionic liquid are more resistive than the pore walls by a factor of 30,000 or so.

In most carbon-based supercapacitors (whether aqueous, organic, or ionic liquid), the solitary RC-parallel network on the right-hand side of Fig. 4 dominates the impedance response at high frequencies $(>1 \mathrm{kHz}$, say) whereas the vertical ladder network dominates the impedance response at low frequencies. In the latter case, the time constants of the "rungs" of the ladder network are typically distributed over many orders of magnitude, from milliseconds to kiloseconds.

Sometimes, an approximately linear segment is observed in the complex plane of impedance at a fixed angle to the real axis. This state of affairs is shown (in idealized form) in Fig. 5. Over the range of frequencies corresponding to the linear segment, the finite ladder network is indistinguishable from a well-known infinite ladder network today known as a "constant phase element" (CPE) [104]. The mathematical form of this element is

$Z=\frac{1}{T(j \omega)^{p}}$

where $Z$ is the complex impedance, $\omega$ is the radial frequency, and $j=\sqrt{-1}$. The terms $T$ and $p$ are constants, and the corresponding phase angle is $(-90 p)$ degrees. In Fig. 5, the fact that the linear segment is not vertical indicates that the capacitances in the infinite ladder network are charging

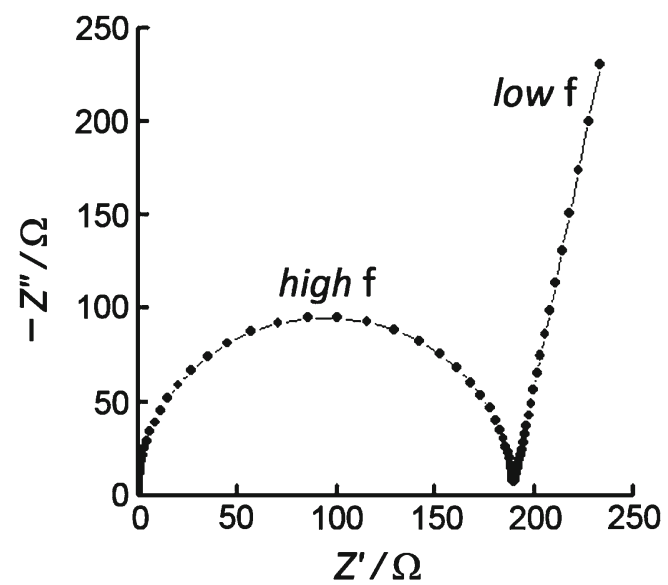

Fig. 5 Complex plane impedance plot of a model circuit consisting of a $\mathrm{CPE}$ in series with an RC-parallel network. Frequency range $=100 \mathrm{~Hz}$ to $1 \mathrm{GHz}, \mathrm{CPE}-\mathrm{T}=1.1 \times 10^{-5} \mathrm{~F}, \mathrm{CPE}-\mathrm{p}=0.87, R=190 \Omega$, and $C=9.4 \times$ $10^{-10} \mathrm{~F}$ asynchronously (i.e. at different rates) such that greater amounts of charge are stored at longer times (lower frequencies).

More realistic than infinite ladder networks are finite ladder networks. These latter behave as single, total capacitances at very long times (i.e. at very low frequencies) having the value

$C_{\text {real }}=\sum_{1}^{\infty} C_{1}+C_{2}+C_{3}+\ldots$

In this limit the complex plane impedance plot takes the form of a vertical straight line, as shown in Fig. 6. As the limit of a sum, the value of $C_{\text {real }}$ is the largest that can be measured on a supercapacitor and therefore tends to be the value quoted by manufacturers. Naturally, it should not be mistaken for the value of equivalent series capacitance that is measured on any finite time scale (and which is necessarily smaller than $C_{\text {real }}$ ).

\section{Comparison of theory and experiment}

The presence of a vertical ladder network in an electrochemical equivalent circuit indicates a distribution of time constants in the real system. Figure 7 shows what a finite ladder network looks like when the rungs of the ladder are ordered in terms of their time constants $\tau_{1}<\tau_{2}<\tau_{3} \ldots$. As time progresses, the capacitors charge sequentially from bottom to top. We call this phenomenon asynchronous charging. It is the existence of asynchronous charging that causes the practical problems of open circuit voltage decay, capacitance loss at high frequency, and voltammetric distortions at high scan rate, as we now describe.

In order to compare theory and experiment, we constructed a supercapacitor simulator from ordinary electrical components using the values indicated in Fig. 8. Its responses were

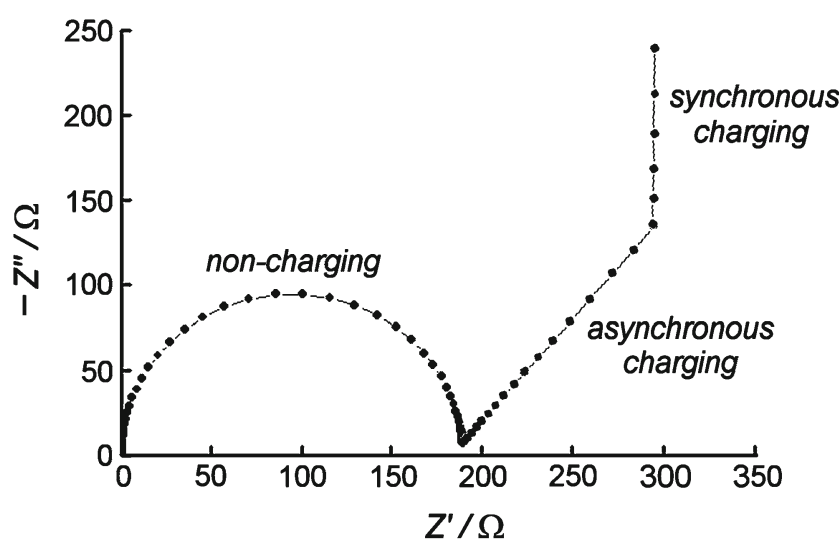

Fig. 6 Complex plane impedance plot of the universal equivalent circuit for a symmetric carbon-based supercapacitor (idealized). In reality, the asynchronous charging region will exhibit a fine structure depending on the distribution of resistance and capacitance values in the electrodes 


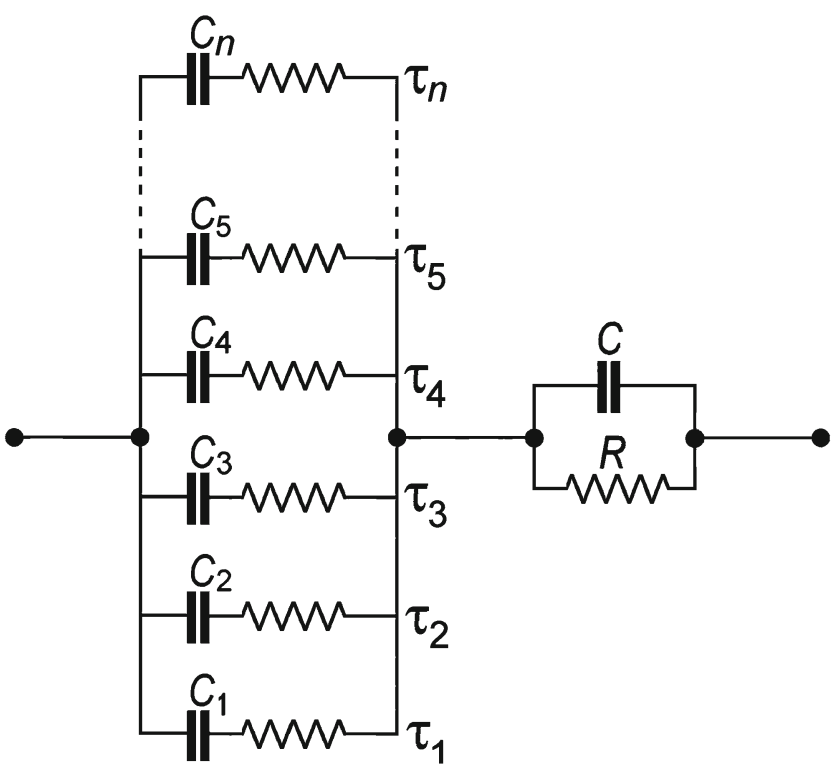

Fig. 7 The finite ladder network of a symmetric supercapacitor, arranged in order of the time constants $\tau_{1}<\tau_{2}<\tau_{3} \ldots$. The capacitors charge in the sequence $C_{1}, C_{2}, C_{3} \ldots$, so that the equivalent series capacitance appears larger at smaller scan rates. [Note: Depending on supercapacitor design, the solution capacitance $C$ may act as a constant phase element.]

then compared with those of a commercial 1-F supercapacitor (Maxwell Technologies BCAP0001, 2.7 V). For simplicity we restricted the simulator to three rungs of the vertical ladder network, and to prevent contributions from contact resistance, we avoided milliohm $(\mathrm{m} \Omega)$ resistors. The time constants of the ladder network were arbitrarily assigned values of $10 \mathrm{~ms}$, $0.1 \mathrm{~s}$, and $1.0 \mathrm{~s}$.

The open circuit voltage decay curves for the supercapacitor simulator, after charging potentiostatically at $2.0 \mathrm{~V}$ for various times, are shown in Fig. 9. The corresponding results for a commercial 1-F supercapacitor are shown in Fig. 10. In the scientific literature, these curves are commonly attributed to "self-discharge" (i.e. the presence of a faradaic leakage current through a path of fixed resistance), although there is scant physical evidence for this [105]. An alternative explanation is that ions might diffuse from regions of high electrochemical

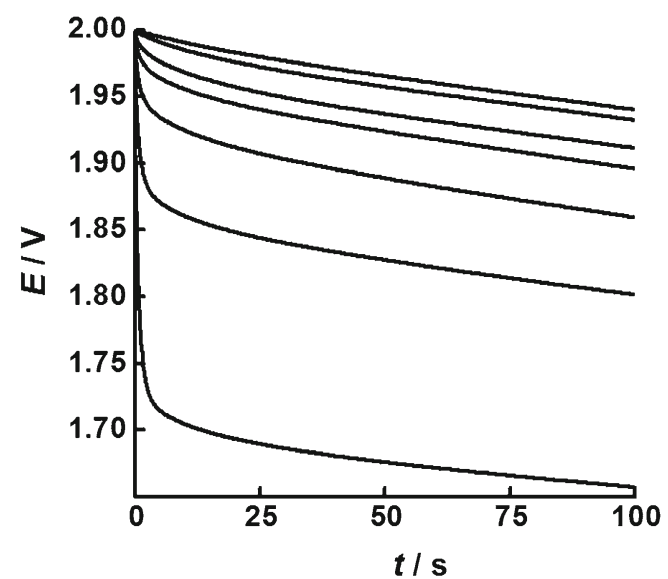

Fig. 9 Open circuit voltage decay of the supercapacitor simulator, after charging potentiostatically at $2.0 \mathrm{~V}$ for various times (top to bottom: 20 , $10,5,4,3,2$, and $1 \mathrm{~s})$. The data indicate that the system is charging in the asynchronous regime

potential to regions of low electrochemical potential [106], but, again, there is little proof. A third suggestion is that the $\mathrm{Fe}(\mathrm{II}) /$ $\mathrm{Fe}(\mathrm{III})$ redox couple might act as a charge shuttle between the terminals of the device [107, 108]. This, however, seems feasible only in aqueous solutions. Finally, a fourth suggestion is that the open circuit voltage decay curves might be due to the spontaneous redistribution of adsorbed ions among states having different RC time constants $[109,110]$. Our ladder network model (Fig. 7) strongly supports this last interpretation. Indeed, at open circuit, it is clear that some fraction of the charge that previously entered the "rapid" states at high potential spontaneously migrates into the "slow" states at low potential, driven by the second law of thermodynamics. During this charge redistribution process, no faradaic reactions occur, and no charge is lost, but some of the electrostatic potential energy of the initially stored charge is necessarily dissipated as heat. Indeed, the production of heat is a natural consequence of the intra-electrode circulation of electric current (Joule heating).

The complex plane impedance plot for the supercapacitor simulator is shown in Fig. 11, and the corresponding result for a commercial 1-F supercapacitor is shown in Fig. 12. The

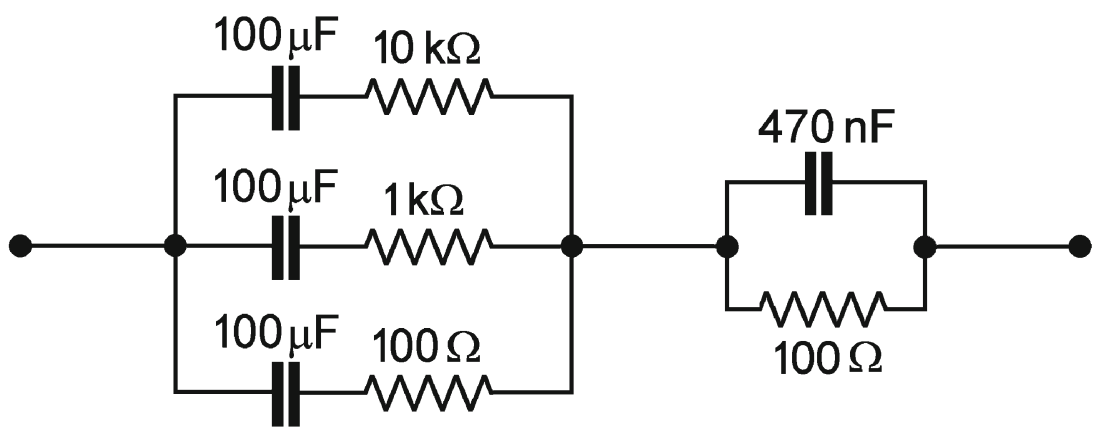

Fig. 8 Component values of a solid-state supercapacitor simulator. This circuit is a truncated version of the universal equivalent circuit having $\mathrm{RC}$ time constants $\tau_{1}=10 \mathrm{~ms}, \tau_{2}=0.1 \mathrm{~s}$, and $\tau_{3}=1 \mathrm{~s}$. As expected, the equivalent series resistance is frequency dependent, approaching a constant value of $90.09 \Omega$ at very high frequencies. Likewise, the equivalent series capacitance is also frequency dependent, approaching a constant value of $300 \mu \mathrm{F}$ at very low frequencies 


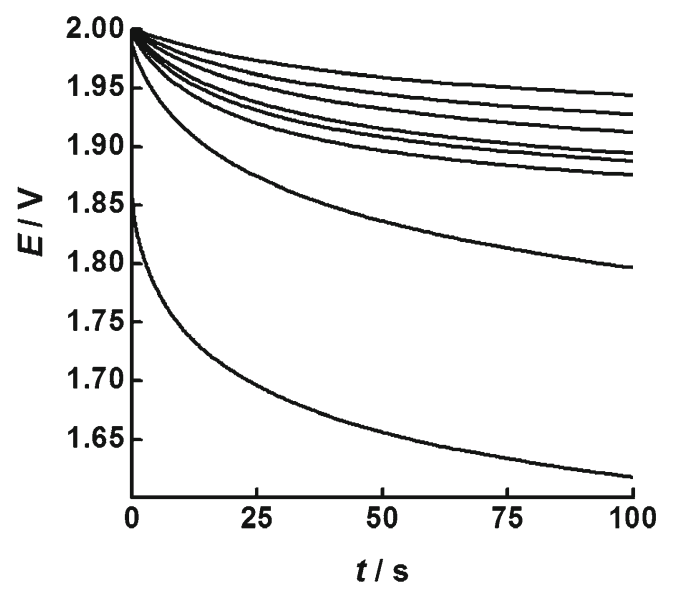

Fig. 10 Open circuit voltage decay of a commercial 1-F supercapacitor (Maxwell Technologies BCAP0001, $2.7 \mathrm{~V}$ ) after charging potentiostatically at $2.0 \mathrm{~V}$ for various times (top to bottom: $30,20,15$, $10,8,6,4$, and $2 \mathrm{~s})$. The data indicate that the system is charging in the asynchronous regime

similarities with Fig. 6 are obvious. In the high frequency range, there is a well-defined arc associated with the resistance of the bulk of solution shunted by its capacitance, which we refer to as the non-charging regime. We chose this name because the carbon electrodes cannot charge in this range of frequencies (although a small amount of charge can of course be stored in the dielectric polarization of the solvent). As commonly observed in many electrochemical systems, the experimental arc is somewhat less than a full semicircle, most likely due to the spatial distribution of $R$ and $C$ values in the bulk of solution. Further, in the low frequency range, there is an approximately linear segment disposed at an angle to the real axis, which corresponds to a set of capacitances that charge and discharge asynchronously. As emphasized above, this segment arises from the wide distribution of time constants of the capacitive charging processes inside the pores.

In the commercial 1-F supercapacitor, we note that the highfrequency response is dominated by the ionic conductivity of the solution outside the pores (i.e. the bulk of solution) whereas the low-frequency response is dominated by the ionic conductivity of the solution inside the pores.

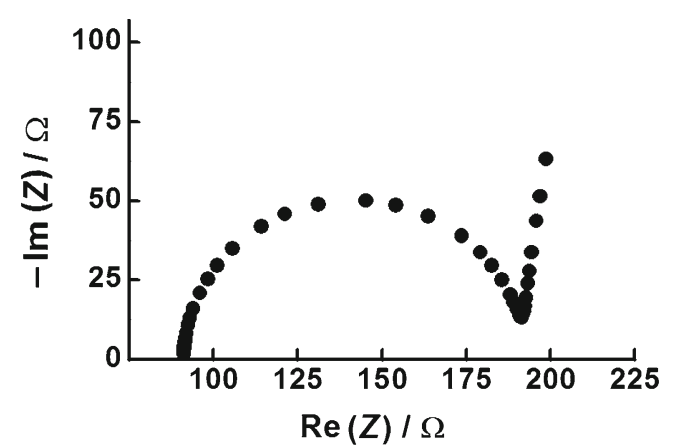

Fig. 11 Complex plane impedance plot for the supercapacitor simulator. The range of frequency measurements was $20 \mathrm{~Hz}$ to $500 \mathrm{kHz}$

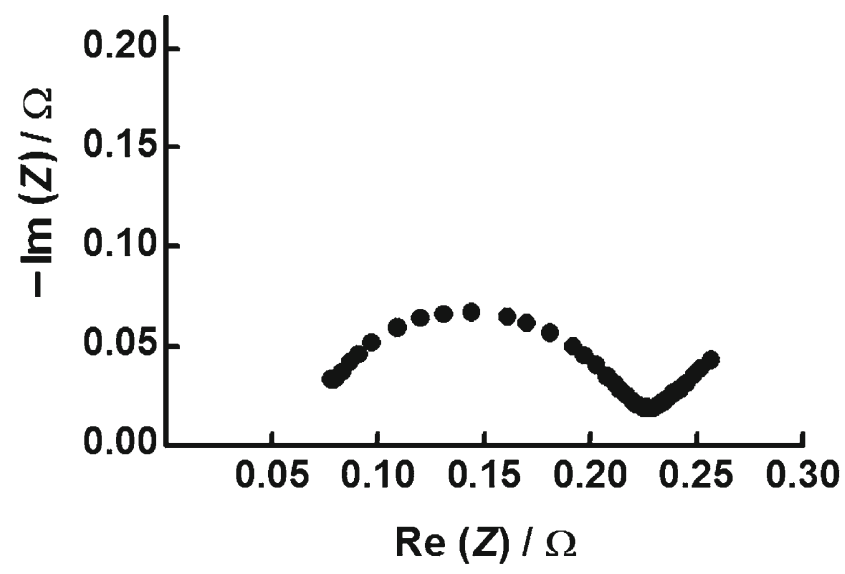

Fig. 12 Complex plane impedance plot for a commercial 1-F supercapacitor (Maxwell Technologies BCAP0001, 2.7 V). The range of frequency measurements was $20 \mathrm{~Hz}$ to $500 \mathrm{kHz}$

Data obtained by applying cyclic voltammetry to the supercapacitor simulator are shown in Fig. 13, and the corresponding data for a commercial 1-F supercapacitor are shown in Fig. 14. In both cases, the low-scan-rate voltammograms have the classic "rounded parallelogram" shape expected for a single RC series circuit. The results indicate that the system is charging and discharging in the synchronous regime. However, with increasing scan rate, the voltammograms become increasingly biconvex (lens-shaped) due to the inability of the slow fraction of capacitive states to "keep up" with the applied voltage perturbation. The data now indicate that the system is charging and discharging in the asynchronous regime. Once again, the universal equivalent circuit (Fig. 4) fully explains the experimental results.

Finally, we remark upon one of the most surprising features of carbon-based supercapacitors, namely the fact that their equivalent series capacitance increases at higher temperatures

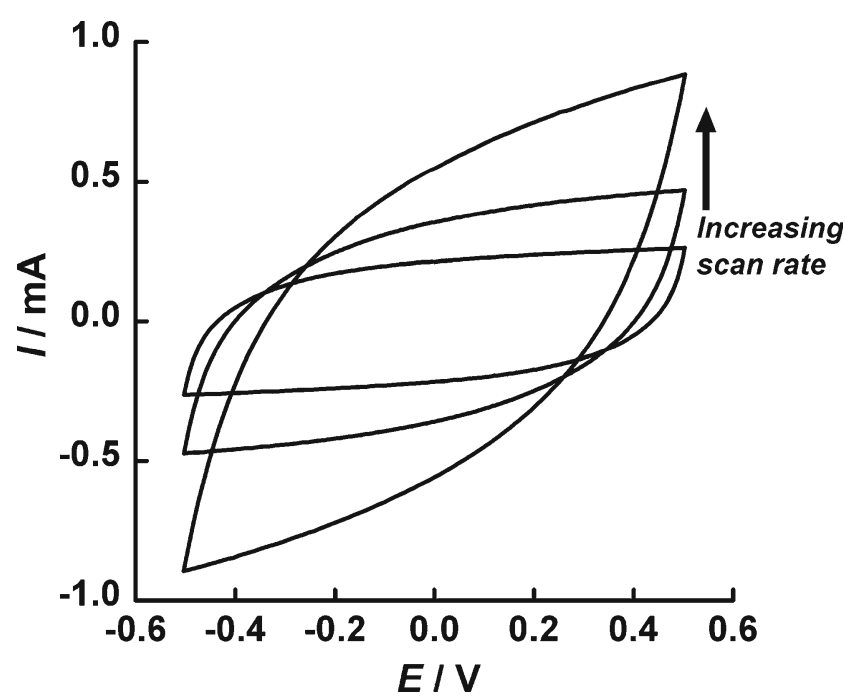

Fig. 13 Cyclic voltammograms of the simulator at increasing scan rates $(1,2$, and $5 \mathrm{~V} / \mathrm{s})$ 


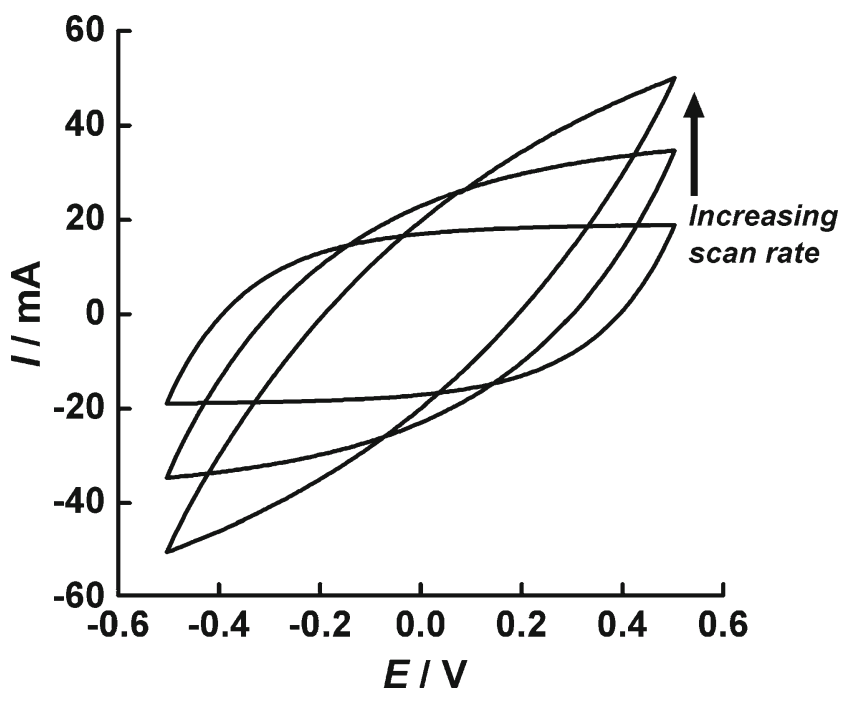

Fig. 14 Cyclic voltammograms of a commercial 1-F supercapacitor (Maxwell Technologies BCAP0001, $2.7 \mathrm{~V}$ ) at increasing scan rates. Electrodes: activated carbon. Electrolyte: tetraethylammonium tetrafluoroborate in acetonitrile. Temperature: $25{ }^{\circ} \mathrm{C}$. Scan rates: 20, 40, and $80 \mathrm{mV} / \mathrm{s}$

(Fig. 15). Since the capacitance of a dielectric liquid generally decreases at higher temperature (because the increased random motion of electrolyte species diminishes the dielectric constant), the observed increase in the case of supercapacitors appears profoundly anomalous. However, the effect can now be explained by reference to the universal equivalent circuit (Fig. 4). As the temperature is raised, the real capacitance actually does diminish (as predicted by the theory of dielectrics) but so too does the real resistance. This combination of effects drives down the RC time constants in the system, so that, on all time scales except the very slowest, the equivalent series capacitance actually increases. Stated simply, more

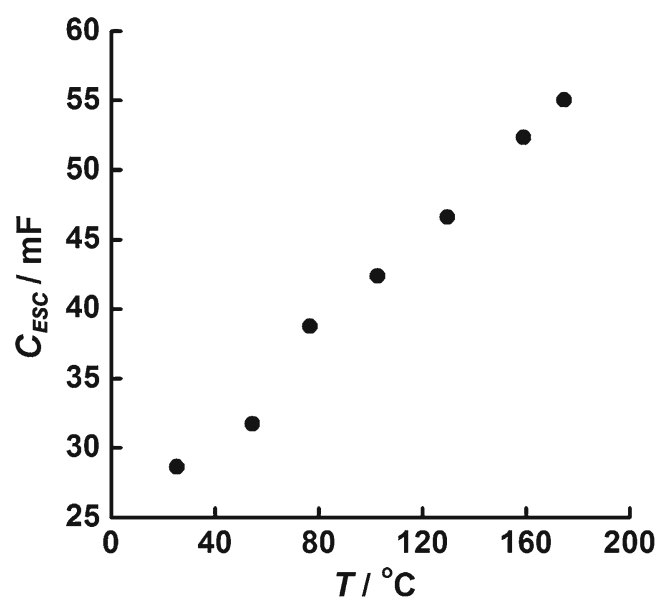

Fig. 15 Equivalent series capacitance versus temperature for a hightemperature ionic liquid-based supercapacitor. Electrodes: activated carbon $\left(100 \mu \mathrm{m} \times 1 \mathrm{~cm}^{2}\right)$. Solvent: butyltrimethylammonium bis(trifluoromethylsulfonyl)imide. Voltammograms recorded at approximately $25{ }^{\circ} \mathrm{C}$ intervals from 25 to $200{ }^{\circ} \mathrm{C}$ at $20 \mathrm{mV} / \mathrm{s}$ equivalent series capacitance is gained by faster charging than is lost by thermal agitation of the solution.

This "temperature paradox" is very striking in ionic liquids because of the steepness of the Vogel-TammannFulcher relation, which controls the solution resistivity. For example, the resistivity of butyltrimethylammonium bis(trifluoromethylsulfonyl)imide (BTM) decreases from $476 \Omega \mathrm{cm}$ at $25{ }^{\circ} \mathrm{C}$ to $17.5 \Omega \mathrm{cm}$ at $200{ }^{\circ} \mathrm{C}$, a factor of nearly 30 [95]. This precipitous fall causes a dramatic lowering of the RC time constants of the pores and results in a doubling of the equivalent series capacitance of hightemperature supercapacitors [95].

\section{Conclusions}

There has long been a need for a definitive equivalent circuit for carbon-based supercapacitors, both to parameterize practical devices and to predict how they will perform under load. In the present work, a universal equivalent circuit has been identified which successfully solves both problems. This circuit explains the three most significant performance-limiting features of present-day devices, namely open circuit voltage decay, capacitance loss at high frequency, and voltammetric distortions at high scan rate. In addition, the circuit reproduces the complex plane impedance behaviour of commercial devices, such as the Maxwell Technologies 1-F supercapacitor, and also reveals why the equivalent series capacitance increases with temperature.

In the scientific literature, the decay of voltage at open circuit has often been attributed to self-discharge (i.e. unwanted faradaic processes). This is, however, an error. In fact, open circuit voltage decay occurs because of the very wide distribution of time constants among the pores in the activated carbon. What happens is that the charge that is initially stored inside quickly-responding pores gradually redistributes itself among slowly-responding pores. At open circuit, this redistribution causes the observed voltage to decrease. Strong evidence in favour of this interpretation is that the observed voltage does not decay asymptotically to zero, but instead decays asymptotically to different finite values depending on the state of charge.

Careful comparison of experimental responses with theoretical predictions reveals the existence of three different regimes in three different ranges of frequency. At high frequency is the non-charging regime, dominated by the behaviour of the bulk of solution. At intermediate frequency is the asynchronous charging regime, dominated by the different $\mathrm{RC}$ time constants of the pores. Finally, at low frequency is the synchronous charging regime, in which all the pores charge simultaneously. At the present time, practical devices tend to operate in the asynchronous charging regime. 
Based on a truncated version of the universal equivalent circuit, a solid-state supercapacitor simulator has also been designed and built, having just three $\mathrm{RC}$ time constants. These time constants were arbitrarily selected to be $\tau_{1}=10 \mathrm{~ms}, \tau_{2}=$ $0.1 \mathrm{~s}$, and $\tau_{3}=1 \mathrm{~s}$, although any suitable values could have been chosen. The resulting device, which is cheap and simple to construct from readily available components, may find practical use among developers of control and switching electronics for supercapacitor storage systems and hybrid electric vehicles. It may also find use as a teaching aid.

It is clear from the present work that a deeper understanding of the electrical properties of nanopores will be essential to the future development of supercapacitors. To date, the minimization of pore resistance has been comparatively neglected in favour of maximizing pore capacitance. However, there is clearly no point in increasing the capacitance of pores if they cannot be charged on any reasonable time scale.

Finally, it is recommended that, in future, researchers in the field of supercapacitors state explicitly which kind of capacitance they are reporting - equivalent series capacitance or real capacitance. Making this distinction would improve the reader's confidence in their data and permit more lucid comparison of results from different laboratories.

Acknowledgments This work was sponsored by the EPSRC (UK) Grant Number EP/I02123X/1 (Battery/Supercapacitor Hybrids for Transport Energy Storage).

\section{References}

1. Conway BE (1999) Electrochemical supercapacitors: scientific fundamentals and technological applications. Kluwer-Plenum, New York

2. Kötz R, Carlen M (2000) Principles and applications of electrochemical capacitors. Electrochim Acta 45:2483-2498

3. Pandolfo AG, Hollenkamp AF (2006) Carbon properties and their role in supercapacitors. J Power Sources 157:11-27

4. Frackowiak E (2007) Carbon materials for supercapacitor application. Phys Chem Chem Phys 9:1774-1785

5. Wang G, Zhang L, Zhang J (2012) A review of electrode materials for electrochemical supercapacitors. Chem Soc Rev 41:797-828

6. Qu DY, Shi H (1998) Studies of activated carbons used in doublelayer capacitors. J Power Sources 74:99-107

7. Gamby J, Taberna PL, Simon P, Fauvarque JF, Chesneau M (2001) Studies and characterisations of various activated carbons used for carbon/carbon supercapacitors. J Power Sources 101:109-116

8. Xue Y, Chen Y, Zhang ML, Yan YD (2008) A new asymmetric supercapacitor based on $\lambda-\mathrm{MnO}_{2}$ and activated carbon electrodes. Mater Lett 62:3884-3886

9. Zhang LL, Zhao XS (2009) Carbon-based materials as supercapacitor electrodes. Chem Soc Rev 38:2520-2531

10. Lin JH, Ko TH, Lin YH, Pan CK (2009) Various treated conditions to prepare porous activated carbon fiber for application in supercapacitor electrodes. Energy Fuels 23:4668-4677

11. Wang HQ, Li ZS, Huang YG, Li QY, Wang XY (2010) A novel hybrid supercapacitor based on spherical activated carbon and spherical $\mathrm{MnO}_{2}$ in a non-aqueous electrolyte. J Mater Chem 20: 3883-3889

12. Barranco V, Lillo-Rodenas MA, Linares-Solano A, Oya A, Pico F, Ibañez J, Agullo-Rueda F, Amarilla JM, Rojo JM (2010) Amorphous carbon nanofibers and their activated carbon nanofibers as supercapacitor electrodes. J Phys Chem C 114:10302-10307

13. Zhang ZJ, Cui P, Chen XY, Liu JW (2013) The production of activated carbon from cation exchange resin for high-performance supercapacitor. J Solid State Electrochem 17:1749-1758

14. Nelson PA, Owen JR (2003) A high-performance supercapacitor/ battery hybrid incorporating templated mesoporous electrodes. J Electrochem Soc 150:A1313-A1317

15. Zhou H, Zhu S, Hibino M, Honma I (2003) Electrochemical capacitance of self-ordered mesoporous carbon. J Power Sources 122: 219-223

16. Vix-Guterl C, Frackowiak E, Jurewicz K, Friebe M, Parmentier J, Béguin F (2005) Electrochemical energy storage in ordered porous carbon materials. Carbon 43:1293-1302

17. Fuertes AB, Lota G, Centeno TA, Frackowiak E (2005) Templated mesoporous carbons for supercapacitor application. Electrochim Acta 50:2799-2805

18. Xing W, Qiao SZ, Ding RG, Li F, Lu GQ, Yan ZF, Cheng HM (2006) Superior electric double layer capacitors using ordered mesoporous carbons. Carbon 44:216-224

19. Xia K, Gao Q, Jiang J, Hu J (2008) Hierarchical porous carbons with controlled micropores and mesopores for supercapacitor electrode materials. Carbon 46:1718-1726

20. Xing W, Huang CC, Zhuo SP, Yuan X, Wang GQ, HulicovaJurcakova D, Yan ZF, Lu GQ (2009) Hierarchical porous carbons with high performance for supercapacitor electrodes. Carbon 47: 1722

21. Ren TZ, Liu L, Zhang Y, Yuan ZY (2013) Nitric acid oxidation of ordered mesoporous carbons for use in electrochemical supercapacitors. J Solid State Electrochem 17:2223-2233

22. Ma X, Liu M, Gan L, Zhao Y, Chen L (2013) Synthesis of microand mesoporous carbon spheres for supercapacitor electrode. J Solid State Electrochem 17:2293-2301

23. Zhu Z, Hu Y, Jiang H, Li C (2014) A three-dimensional ordered mesoporous carbon/carbon nanotubes nanocomposites for supercapacitors. J Power Sources 246:402-408

24. Toupin M, Bélanger D, Hill IR, Quinn D (2005) Performance of experimental carbon blacks in aqueous supercapacitors. J Power Sources 140:203-210

25. Zhu M, Weber CJ, Yang Y, Konuma M, Starke U, Kern K, Bittner AM (2008) Chemical and electrochemical ageing of carbon materials used in supercapacitor electrodes. Carbon 46:1829-1840

26. Jacob GM, Yang QM, Zhitomirsky I (2009) Electrodes for electrochemical supercapacitors. Mater Manuf Process 24:1359-1364

27. Kossyrev P (2012) Carbon black supercapacitors employing thin electrodes. J Power Sources 201:347-352

28. Miller JM, Dunn B, Tran TD, Pekala RW (1997) Deposition of ruthenium nanoparticles on carbon aerogels for high energy density supercapacitor electrodes. J Electrochem Soc 144:L309-L311

29. Wang J, Zhang SQ, Guo YZ, Shen J, Attia SM, Zhou B, Zheng GZ, Gui YS (2001) Morphological effects on the electrical and electrochemical properties of carbon aerogels. J Electrochem Soc 148: D75-D77

30. Li J, Wang X, Huang Q, Gamboa S, Sebastian PJ (2006) Studies on preparation and performances of carbon aerogel electrodes for the application of supercapacitor. J Power Sources 158:784-788

31. Wang X, Liu L, Wang X, Bai L, Wu H, Zhang X, Yi L, Chen Q (2011) Preparation and performances of carbon aerogel microspheres for the application of supercapacitor. J Solid State Electrochem 15:643-648

32. Qian H, Kucernak AR, Greenhalgh ES, Bismarck A, Shaffer MSP (2013) Multifunctional structural supercapacitor composites based 
on carbon aerogel modified high performance carbon fiber fabric. ACS Appl Mater Interfaces 5:6113-6122

33. Niu C, Sichel EK, Hoch R, Moy D, Tennent H (1997) High power electrochemical capacitors based on carbon nanotube electrodes. Appl Phys Lett 70:1480-1482

34. Frackowiak E, Metenier K, Bertagna V, Beguin F (2000) Supercapacitor electrodes from multiwalled carbon nanotubes. Appl Phys Lett 77:2421-2423

35. An KH, Kim WS, Park YS, Choi YC, Lee SM, Chung DC, Bae DJ, Lim SC, Lee YH (2001) Supercapacitors using single-walled carbon nanotube electrodes. Adv Mater 13:497-500

36. An KH, Kim WS, Park YS, Moon JM, Bae DJ, Lim SC, Lee YS, Lee YH (2001) Electrochemical properties of high-power supercapacitors using single-walled carbon nanotube electrodes. Adv Funct Mater 11:387-392

37. Frackowiak E, Béguin F (2002) Electrochemical storage of energy in carbon nanotubes and nanostructured carbons. Carbon 40:17751787

38. AN KH, Jeon KK, Heo JK, Lim SC, Bae DJ, Lee YH (2002) Highcapacitance supercapacitor using a nanocomposite electrode of single-walled carbon nanotube and polypyrrole. J Electrochem Soc 149:A1058-A1068

39. Kim C, Yang KS (2003) Electrochemical properties of carbon nanofiber web as an electrode for supercapacitor prepared by electrospinning. Appl Phys Lett 83:1216-1218

40. Picó F, Rojo JM, Sanjuan ML, Anson A, Benito AM, Callejas MA, Maser WK, Martinez MT (2004) Single-walled carbon nanotubes as electrodes in supercapacitors. J Electrochem Soc 151:A831-A837

41. Yoon BJ, Jeong SH, Lee KH, Kim HS, Park CG, Han JH (2004) Electrical properties of electrical double layer capacitors with integrated carbon nanotube electrodes. Chem Phys Lett 388:170-174

42. Du C, Yeh J, Pan N (2005) High power density supercapacitors using locally aligned carbon nanotube electrodes. Nanotechnology $16: 350-353$

43. Du C, Pan N (2006) High power density supercapacitor electrodes of carbon nanotube films by electrophoretic deposition. Nanotechnology 17:5314-5318

44. Yang C, Kim Y, Endo M, Kanoh H, Yudasaka M, Iijima S, Kaneko K (2007) Nanowindow-regulated specific capacitance of supercapacitor electrodes of single-wall carbon nanohorns. J Am Chem Soc 129:20-21

45. Yan X, Tai Z, Chen J, Xue Q (2011) Fabrication of carbon nanofiber-polyaniline composite flexible paper for supercapacitor. Nanoscale 3:212-216

46. Kim BH, Yang KS, Kim YA, Kim YJ, An B, Oshida K (2011) Solvent-induced porosity control of carbon nanofiber webs for supercapacitor. J Power Sources 196:10496-10501

47. Wang Y, Shi Z, Huang Y, Ma Y, Wang C, Chen M, Chen Y (2009) Supercapacitor devices based on graphene materials. J Phys Chem C 113:13103-13107

48. Zhang K, Zhang LL, Zhao XS, Wu J (2010) Graphene/polyaniline nanofiber composites as supercapacitor electrodes. Chem Mater 22: 1392-1401

49. Liu C, Yu Z, Neff D, Zhamu A, Jang BZ (2010) Graphene-based supercapacitor with an ultrahigh energy density. Nano Lett 10 : 4863-4868

50. Yan J, Fan Z, Wei T, Qian W, Zhang M, Wei F (2010) Fast and reversible surface redox reaction of graphene- $\mathrm{MnO}_{2}$ composites as supercapacitor electrodes. Carbon 48:3825-3833

51. Zhang LL, Zhou R, Zhao XS (2010) Graphene-based materials as supercapacitor electrodes. J Mater Chem 20:5983-5992

52. Biswas S, Drzal LT (2010) Multilayered nanoarchitecture of graphene nanosheets and polypyrrole nanowires for high performance supercapacitor electrodes. Chem Mater 22(20):5667-5671

53. Lake J, Tanaka Z, Chen B (2012) Graphene composite materials for supercapacitor electrodes. MRS Proc 1407:141-147
54. Zhang S, Li Y, Pan N (2012) Graphene based supercapacitor fabricated by vacuum filtration deposition. J Power Sources 206:476482

55. Ye S, Feng J, Wu P (2013) Deposition of three-dimensional graphene aerogel on nickel foam as a binder-free supercapacitor electrode. ACS Appl Mater Interfaces 5:7122-7129

56. Lu Y, Zhang F, Zhang T, Leng K, Zhang L, Yang X, Ma Y, Huang Y, Zhang M, Chen Y (2013) Synthesis and supercapacitor performance studies of $\mathrm{N}$-doped graphene materials using ophenylenediamine as the double-N precursor. Carbon 63:508-516

57. Zubieta L, Bonert R (2000) Characterization of double-layer capacitors for power electronics applications. IEEE Trans Ind Appl 36: 199-205

58. Jurewicz K, Delpeux S, Bertagna V, Beguin F, Frackowiak E (2001) Supercapacitors from nanotubes/polypyrrole composites. Chem Phys Lett 347:36-40

59. Taberna PL, Simon P, Fauvarque JF (2003) Electrochemical characteristics and impedance spectroscopy studies of carbon-carbon supercapacitors. J Electrochem Soc 150:A292-A300

60. Buller S, Thele M, De Doncker RW, Karden E (2003). Impedancebased simulation models of supercapacitors and $\mathrm{Li}$-ion batteries for power electronic applications. In: Conference record of the IEEE industry applications conference, 38th IAS annual meeting. (Vol. 3, pp. 1596-1600), IEEE, Piscataway

61. Kötz R, Hahn M, Gallay R (2006) Temperature behavior and impedance fundamentals of supercapacitors. J Power Sources 154: $550-555$

62. Wei T, Qi X, Qi Z (2007) An improved ultracapacitor equivalent circuit model for the design of energy storage power systems. In: Proceedings of the international conference on electrical machines and systems, 8-11 Oct 2007, Seoul, pp 69-73

63. Lisheng S, Crow ML (2008) Comparison of ultracapacitor electric circuit models. In: Power and Energy Society general meeting conversion and delivery of electrical energy in the 21 st century, 20 24 July 2008, Pittsburgh, pp 1-6

64. Li Z, Chen J (2008) An impedance-based approach to predict the state-of-charge for carbon-based supercapacitors. Microelectron Eng 85:1549-1554

65. Bakhmatyuk BP, Venhryn BY, Grygorchak II, Micov MM (2008) Influence of chemical modification of activated carbon surface on characteristics of supercapacitors. J Power Sources 180:890-895

66. Lockett V, Sedev R, Ralston J, Horne M, Rodopoulos T (2008) Differential capacitance of the electrical double layer in imidazolium-based ionic liquids: influence of potential, cation size, and temperature. J Phys Chem C 112:7486-7495

67. Li K, Zhu C, Lu R, Chan CC (2012) The improved study of thermal dependence equivalent circuit model for supercapacitor. In: 16th international symposium on electromagnetic launch technology, 15-19 May 2012, Beijing, pp 1-5

68. Basnayaka PA, Ram MK, Stefanakos L, Kumar A (2013) Graphene/polypyrrole nanocomposite as electrochemical supercapacitor electrode: electrochemical impedance studies. Graphene 2:81-87

69. Kim T, Jung G, Yoo S, Suh KS, Ruoff RS (2013) Activated graphene-based carbons as supercapacitor electrodes with macroand mesopores. ACS Nano 7:6899-6905

70. Musolino V, Piegari L, Tiron E (2013) New full frequency range supercapacitor model with easy identification procedure. IEEE Trans Ind Electron 60:112-120

71. Pell WG, Conway BE (2001) Analysis of power limitations at porous supercapacitor electrodes under cyclic voltammetry modulation and dc charge. J Power Sources 96:57-67

72. Pell WG, Conway BE (2001) Voltammetry at a de Levie brush electrode as a model for electrochemical supercapacitor behaviour. J Electroanal Chem 500:121-133

73. Kimizuka O, Tanaike O, Yamashita Hiraoka JT, Futaba D, Hata K, 
Machida K, Suematsu S, Tamamitsu K, Saeki S, Yamada Y, Hatori H (2008) Electrochemical doping of pure single-walled carbon nanotubes used as supercapacitor electrodes. Carbon 46:1999-2001

74. Yu D, Dai L (2009) Self-assembled graphene/carbon nanotube hybrid films for supercapacitors. J Phys Chem Lett 1:467-470

75. Li ZJ, Yang BC, Zhang SR, Zhao CM (2012) Graphene oxide with improved electrical conductivity for supercapacitor electrodes. Appl Surf Sci 258:3726-3731

76. Sun J, He L, Zhao Q, Cai L, Song R, Hao Y, Ma Z, Huang W (2012) A simple and controllable nanostructure comprising non-conductive poly(vinylidene fluoride) and graphene nanosheets for supercapacitor. Front Mater Sci 6:149-159

77. Tashima D, Kishita T, Maeno S, Nagasawa Y (2013) Mesoporous graphitized Ketjenblack as conductive nanofiller for supercapacitors. Mater Lett 110:105-107

78. Kornyshev AA (2007) Double-layer in ionic liquids: paradigm change? J Phys Chem B 111:5545-5557

79. Huang J, Sumpter BG, Meunier V (2008) Theoretical model for nanoporous carbon supercapacitors. Angew Chem Int Ed 47:520-524

80. Huang J, Sumpter BG, Meunier V (2008) A universal model for nanoporous carbon supercapacitors applicable to diverse pore regimes, carbon materials, and electrolytes. Chem Eur J 14:6614-6626

81. Fedorov MV, Kornyshev AA (2008) Ionic liquid near a charged wall: structure and capacitance of electrical double layer. J Phys Chem B 112:11868-11872

82. Shim Y, Kim HJ (2010) Nanoporous carbon supercapacitors in an ionic liquid: a computer simulation study. ACS Nano 4:2345-2355

83. Lazzari M, Mastragostino M, Pandolfo AG, Ruiz V, Soavi F (2011) Role of carbon porosity and ion size in the development of ionic liquid based supercapacitors. J Electrochem Soc 158:A22-A25

84. Merlet C, Rotenberg B, Madden PA, Taberna P-L, Simon P, Gogotsi $Y$, Salanne M (2012) On the molecular origin of supercapacitance in nanoporous carbon electrodes. Nat Mater 11:306-310

85. Rajput NN, Monk J, Singh R, Hung FR (2012) On the influence of pore size and pore loading on structural and dynamical heterogeneities of an ionic liquid confined in a slit nanopore. J Phys Chem C 116:5169-5181

86. Jiang D-E, Jin Z, Henderson D, Wu J (2012) Solvent effect on the pore-size dependence of an organic electrolyte supercapacitor. J Phys Chem Lett 3:1727-1731

87. Vatamanu J, Borodin O, Bedrov D (2012) Molecular dynamics simulation study of the interfacial structure and differential capacitance of alkylimidazolium bis(trifluoromethanesulfonyl)imide $\left[\mathrm{C}_{\mathrm{n}} \operatorname{mim}\right][\mathrm{TFSI}]$ ionic liquids at graphite electrodes. J Phys Chem C 116:7940-7951

88. Jiang D-E, Wu J (2013) Microscopic insights into the electrochemical behavior of nonaqueous electrolytes in electric double-layer capacitors. J Phys Chem Lett 4:1260-1267

89. Kondrat S, Kornyshev AA (2013) Charging dynamics and optimization of nano-porous supercapacitors. J Phys Chem C 117:12399-12406

90. Vatamanu J, Hu Z, Bedrov D, Perez C, Gogotsi Y (2013) Increasing energy storage in electrochemical capacitors with ionic liquid electrolytes and nanostructured carbon electrodes. J Phys Chem Lett 4: 2829-2837

91. Fletcher S (1994) Tables of degenerate electrical networks for use in the equivalent circuit analysis of electrochemical systems. J Electrochem Soc 141:1823-1826

92. Lvovich VF (2012) Impedance spectroscopy: applications to electrochemical and dielectric phenomena. Wiley, Hoboken

93. Barsoukov E, Macdonald JR (eds) (2005) Impedance spectroscopy: theory, experiment, and applications. Wiley, Hoboken

94. Orazem ME, Tribollet B (2008) Electrochemical impedance spectroscopy (ECS Series Vol. 48). Wiley, Hoboken

95. Fletcher S, Black VJ, Kirkpatrick I, Varley TS (2013) Quantum design of ionic liquids for extreme chemical inertness and a new theory of the glass transition. J Solid State Electrochem 17:327-337

96. de Levie R (1963) On porous electrodes in electrolyte solutions: I. Capacitance effects. Electrochim Acta 8:751-780

97. de Levie R (1964) On porous electrodes in electrolyte solutions: IV. Electrochim Acta 9:1231-1245

98. Fritts DH (1997) An analysis of electrochemical capacitors. J Electrochem Soc 144:2233-2241

99. Johnson AM, Newman J (1971) Desalting by means of porous carbon electrodes. J Electrochem Soc 118:510-517

100. Posey FA, Morozumi T (1966) Theory of potentiostatic and galvanostatic charging of the double layer in porous electrodes. J Electrochem Soc 113:176-184

101. Fletcher S (1993) Contribution to the theory of conducting-polymer electrodes in electrolyte solutions. J Chem Soc Faraday Trans 89: 311-320

102. Bisquert $\mathrm{J}$ (2002) Theory of the impedance of electron diffusion and recombination in a thin layer. J Phys Chem B 106:325-333

103. Macdonald DD (2009) Why electrochemical impedance spectroscopy is the ultimate tool in mechanistic analysis. Electrochem Soc Trans 19:55-79

104. Cole KS, Cole RH (1941) Dispersion and absorption in dielectrics I. Alternating current characteristics. J Chem Phys 9:341-351

105. Lewandowski A, Jakóbczyk P, Galiński M, Biegun M (2013) Selfdischarge of electrochemical double layer capacitors. Phys Chem Chem Phys 15:8692-8699

106. Ricketts BW, Ton-That C (2000) Self-discharge of carbonbased supercapacitors with organic electrolytes. J Power Sources 89:64-69

107. Kazaryan SA, Kharisov GG, Litvinenko SV, Kogan VI (2007) Selfdischarge related to iron ions and its effect on the parameters of HES $\mathrm{PbO}_{2}\left|\mathrm{H}_{2} \mathrm{SO}_{4}\right| \mathrm{C}$ systems. J Electrochem Soc 154:A751-A759

108. Andreas HA, Lussier K, Oickle AM (2009) Effect of Fecontamination on rate of self-discharge in carbon-based aqueous electrochemical capacitors. J Power Sources 187:275-283

109. Kaus M, Kowal J, Sauer DU (2010) Modelling the effects of charge redistribution during self-discharge of supercapacitors. Electrochim Acta 55:7516-7523

110. Graydon JW, Panjehshahi M, Kirk DW (2014) Charge redistribution and ionic mobility in the micropores of supercapacitors. J Power Sources 245:822-829 